\title{
Median Filter based Wavelet Transform for Multilevel Noise
}

\author{
H S Shukla \\ Department of Computer \\ Science \\ Deen Dayal Upadhaya \\ Gorakhpur \\ University Gorakhpur(UP) \\ India
}

\author{
Narendra Kumar \\ Department of Computer \\ Science \\ Deen Dayal Upadhaya \\ Gorakhpur \\ University Gorakhpur(UP) \\ India
}

\author{
R P Tripathi \\ Department of mathematics \\ GraphicEra University \\ Dehradun India
}

\begin{abstract}
In digital image different kinds of noises exist in an image and a variety of noise reduction techniques are available to perform de-noising. Selection of the de-noising algorithm depends on the types of noise. Gaussian noise, speckle noise, salt \& pepper noise, shot noise are types of noises that are present in an image. The principle approach of image denoising is filtering. Available filters to de-noise an image are median filter, Gaussian filter, average filter, wiener filter and many more. A particular noise can be de-noising by specific filter but multilevel noise are challenging task for digital image processing. In this paper we propose a median filter based Wavelet transform for image de-noising. This technique is used for multilevel noise. In this paper three noise model Gaussian noise, Poisson noise and salt and pepper noise for multilevel noise have been used. In the end of paper we compare our technique with many other de-noise techniques.
\end{abstract}

\section{Keywords}

Gaussian noise, Multilevel noise, Threshold, Wavelet transform, Threshold ratio, Poisson nois

\section{INTRODUCTION}

Noisy image due to errors in the image acquisition process so that pixel values do not reflect the true intensities of actual picture. The presence of noise gives an image with blur and snowy appearance. Wavelets transforms are based on small waves, called wavelet, of varying frequency in limited duration. Wavelet thresholding methods are used for noise removal, in which the wavelet coefficients are thresholded in order to remove their noisy part, Wavelet thresholding methods first time introduced by Donoho in 1993. Wavelet thresholding methods do not require any particular assumptions about the nature of the signal and exploits the spatially adaptive multi-resolution of the wavelet transform. Before sometimes wavelet transforms used in signal and image processing, especially in the field of signal denoising. Donoho et. al [1-5].In the 1990s, the field was dominated by wavelet shrinkage and wavelet thresholding methods .Various methods are available[6].

\section{NOISE MODEL}

The noise model is spatial invariant, i.e., independent of spatial location. The noise model is uncorrelated with the object function. Noise models [7] can be categorized into two groups: additive noise and multiplicative noise.

(a)Additive noise models: In additive noise model, the noise is superimposed upon the image, which resulted in variation of the image signal. Some common noise distributions are

Gaussian noise distribution: Gaussian noise is a statistical noise. It is distributed over the signal. The probability density function (PDF) of Gaussian noise is equal to that of the normal distribution, also known as Gaussian Distribution. $\mathrm{PDF}$ of a Gaussian random variable, $\mathrm{z}$ is given by

$$
p(z)=\frac{1}{\sqrt{2 \pi \sigma}} e^{-\frac{(z-\mu)^{2}}{2 \sigma^{2}}}
$$

Where:

$$
\begin{aligned}
& \mathrm{z}=\text { gray level. } \\
& \mu=\text { mean } \\
& \sigma=\text { standard deviation } \\
& \mathrm{p}(\mathrm{z})=\text { probability density function }
\end{aligned}
$$

Rayleigh noise distribution: The Rayleigh distribution of the probabilities of the random variable $X$ is characterized by the probability density function

$$
f(p)= \begin{cases}\left(p / \sigma^{2}\right) \exp \left(-p / 2 \sigma^{2}\right) & , \text { when } \mathrm{p} \geq 0 \\ 0 & , \text { when } \mathrm{p}<0\end{cases}
$$

The distribution function is

$$
F(x)=\int_{0}^{x} f(p) d p=1-\exp \left(-x^{2} / 2 \sigma^{2}\right)
$$

The mathematical expectation is $E X=\sqrt{\pi / 2 \sigma^{2}}$ and the variance is $D X=(4-\pi) \sigma^{4} / 2$. The maximum value of the density function is equal to $1 / \sigma \sqrt{e}$ and is reached when $x=$ $\sigma$.

Gamma $(a, b)$ noise distribution: PDF of a Gamma(a,b) noise distribution random variable, $\mathrm{z}$ is given by

$$
p(z)=\frac{a^{b} z^{b-1}}{b-1} e^{-a z} \quad, \text { for } z \geq 0 .
$$

where the parameter are such that $a>0, b$ is positive integer ,and " $L$ " indicates factorial. The mean and variance of this density are given by

$$
\bar{z}=\frac{b}{a} \quad \text { and } \quad \sigma^{2}=\frac{b}{a}
$$

Exponential noise distribution: PDF of Exponential noise distribution is

$$
p(z)=a e^{-a z} \quad, \text { for } \mathrm{z} \geq 0 .
$$


Where a $>0$.The mean and variance of this density function are

$$
\begin{aligned}
\bar{z} & =\frac{1}{a} \\
\text { and } \quad \sigma^{2} & =\frac{b}{a}
\end{aligned}
$$

$\mathrm{p}(\mathrm{z})=$ probability density function

$\mathrm{z}=$ gray level.

\section{(b)Multiplicative Noise Models}

In this model the noise is signal dependent, and is multiplied to the image. Two commonly multiplicative noise models are:

Salt-and-Pepper (impulse): The salt-and-pepper noise are also called shot noise, impulse noise or spike noise that is usually caused by faulty memory locations,malfunctioning pixel elements in the camera sensors, or there can be timing errors in the process of digitization .In the salt and pepper noise there are only two possible values exists that is a and $b$ and the probability of each is less than 0.2.If the numbers greater than this numbers the noise will swamp out image. For 8-bit image the typical value for 255 for salt-noise and pepper noise is 0 . Probability density function of impulse noise is given by

$$
p(z)= \begin{cases}p_{a} & , \text { for } \mathrm{z}=\mathrm{a} \\ p_{b} & , \text { for } \mathrm{z}=\mathrm{b} \\ 0 & , \text { otheerwise }\end{cases}
$$

If $\mathrm{b}>\mathrm{a}$, intensity $\mathrm{b}$ will appear as a light dot in the image. level a will appear like a dark dot, if either $p_{a}$ and $p_{b}$ is zero, the impulse noise is called unipolar. If neither probability is zero, and especially if they are approximately equal ,impulse noise values will resemble salt and pepper granules randomly distributed over the image. For this reason, bipolar impulse noise also is called salt and pepper noise.

Speckle noise

$$
a=a_{R}+j a_{l}
$$

Where $a_{R}, a_{I}$ are independent Gaussian, with zero mean Poisson Noise

Poisson noise provides a noise source whose probability density function is not continuous. For example, a random number that can take on only discrete values has a probability function that also is discrete.

\section{FILTER}

If only the noise is presented in the digital image [8], i.e., without considering the degradation function, following techniques can be used to reduce the noise effect:

Mean filter: For every pixel in the image, the pixel value is replaced by the mean value of its neighboring pixels $(N x M)$ with a weight $w_{k}=1 /(N M)$. This will resulted in a smoothing effect in the image.

Median filter: For every pixel in the image, the pixel value is replaced by the statistical median of its neighboring pixels $(N x M)$. Although median filter also provides a smoothing effect, it is better in preserving detailed image information, for example: edges.

Homomorphism filter: In the case of multiplicative noise, one cannot simply apply smooth filter to the observed noisy image $f(x, y)$, as the Fourier transform of the product of two functions is not separable. To overcome this issue, a logarithmic representation of the image model is used instead, i.e.

$$
\operatorname{In}(f(x, y)=\operatorname{In}(o(x, y))+\operatorname{In}(n(x, y))
$$

Where the Fourier transforms of the logarithmic function is

$$
P S N R=10 \log _{10}\left(R^{2} / M S E\right)
$$

Since the noise model, through logarithmic operation, becomes additive, a smooth filter can thus be applied to remove the noise effect.

\section{PARAMETRIC DESCRIPTION}

The parameters considered for image processing are peak signal to noise ratio (PSNR) and mean square error (MSE)

$$
\text { . } P S N R=10 \log _{10}\left(R^{2} / M S E\right)
$$

Where $\mathrm{R}$ is maximum value of the pixel present in an image and MSE is mean square error between the original and denoised image with size $\mathrm{A} * \mathrm{~B}$. Mean square error is defined as:

$$
M S E=\frac{1}{A^{*} B} \sum_{i=1}^{M} \sum_{j=1}^{N}[x(i, j)-y(i, j)]^{2}
$$

Where, $(i$,$) is original image and y(i$,$) is de-noised$ image. Root mean square error is defined as:

$R M S E=s q r t(M S E)$ i.e. root mean square error is square root value of mean square error.

\section{PROPOSED SYSTEM}

\section{Multilevel Noise:}

The noise considered in this paper is a multilevel noise which is combination of Gaussian noise, Salt \& Pepper noise and Poisson noise. Thus the reduction of multilevel becomes an important aspect in the application of digital image. In the first level noisy image is given as

$\mathrm{X}(\mathrm{k}, \mathrm{l})=\mathrm{O}(\mathrm{k}, \mathrm{l}) * \mathrm{G}(\mathrm{k}, \mathrm{l})$

Where $\mathrm{G}$ is Gaussian noise, $\mathrm{O}$ is original image and $\mathrm{X}$ is the noisy image with Gaussian noise. $(\mathrm{k}, \mathrm{l})$ are the variable of spatial location $(\mathrm{k}$ represents the raw and 1 represents the column).

In the second level we are using Gaussian noisy image as an input image in Poisson noise.

$\mathrm{Y}(\mathrm{k}, \mathrm{l})=\mathrm{X}(\mathrm{k}, \mathrm{l}) * \mathrm{P}(\mathrm{k}, \mathrm{l})$

Where $\mathrm{Y}$ is noisy image associated with Gaussian and Poisson noise. $\mathrm{X}$ is a Gaussian noisy image and $\mathrm{P}$ is a Poisson noisy image. $(k, 1)$ are the variable of spatial location ( $k$ represents the raw and 1 represents the column).

In the third level we are using previous level output noisy image as input to $\mathrm{Z}$ image.

$\mathrm{Z}(\mathrm{k}, \mathrm{l})=\mathrm{Y}(\mathrm{k}, \mathrm{l}) * \mathrm{~S}(\mathrm{k}, \mathrm{l})$

Where $\mathrm{Z}$ is the output image with Gaussian noise, Poisson noise Salt \& Pepper noise. Y is the final noisy image output. 
Wavelet transforms using median filter: Wavelet transformation is used for reduction of noise but if we used it for multilevel noise not give better result.

Median filter is a nonlinear filter during median filtering first sorting all the pixel values from the surrounding neighborhood into numerical order and then replacing the pixel being considered with middle pixel value. Median value must be written to a separate array or buffer .Median filter replace the value of a image pixel by the median of intensity level in the neighborhood of that pixel.

$$
f(x, y)=\stackrel{\operatorname{median}\{Z(k, l)\}}{(k, l) \in S_{x y}}
$$

Values of the pixel at $(\mathrm{x}, \mathrm{y})$ is included in the computation of the median, pixel in region defined by $S_{x y}$.

Hard thresholding in wavelet bases: $f$ is the output image after median filtering. efficient non linear de-noising estimator is obtained by threshholding the coefficient of $\mathrm{f}$, which is selected by orthogaonal basis

$$
B=\left\{\psi_{m}\right\} m \text { of } \mathrm{R}^{\mathrm{N}}
$$

De-noise the piecewise regular images on basis of wavelet. The hard thresholding operator with threshold $\mathrm{T} \geq 0$ applied to same image $f$ is defined as

$S_{T}^{0}(f)=\sum_{\left|<f, \psi_{m}\right\rangle>T}<f, \psi_{m}>\psi_{m}=S_{T}^{0}\left(<f, \psi_{m}\right) \psi_{m}$

Where hard threshold operator is

$S_{T}^{0}(\alpha)= \begin{cases}\alpha & ,|\alpha|>T \\ 0 & , \text { otherwise }\end{cases}$

The de-noise estimator is then defined as

$f=S_{T}{ }^{0}(f)$

Wavelet denoising with soft thresholding the estimated image f using hard thresholding.

It is possible to improve the result by soft thresholding defined as

$F=S_{T}^{1}(f)=\sum_{m} S_{T}^{1}\left(\left(f, \psi_{m}\right)\right) \psi_{m}$

Where $S_{T}{ }^{1}(\alpha)=\max \left(0,1-\frac{T}{|\alpha|}\right) \alpha$

$S_{T}{ }^{1}(\alpha)$ is soft thresholding function

Proposed Procedure: to remove the noise of image by increasing the PSNR and MSE value Fig 1 explain the proposed concept. The noise used multilevel which is leveled by Gaussian noise, passion noise and salt $\&$ pepper noise and for filtering applied median filter for the multilevel noise in initial filtering and then applied wavelet transform, hard thresholding and soft thresholding

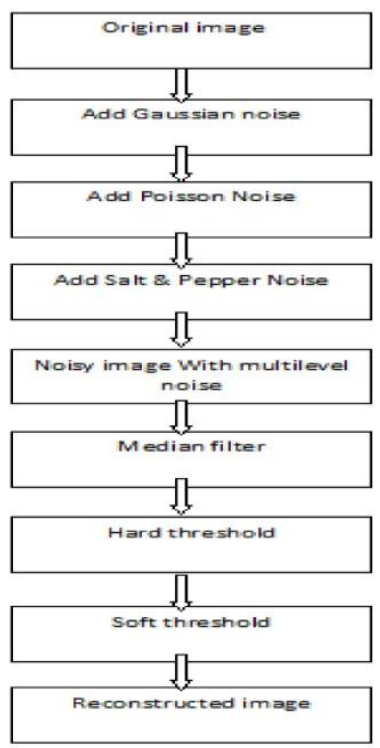

Figure 1: Image reconstruction process using median and wavelet transform

\section{RESULT}

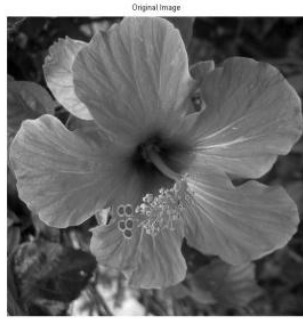

a
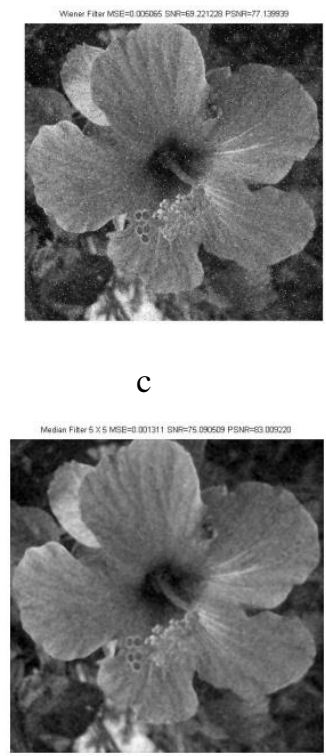

e

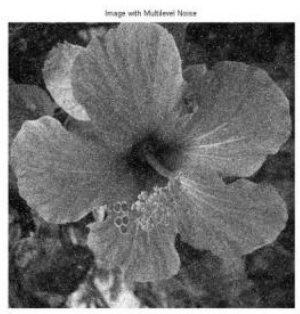

b

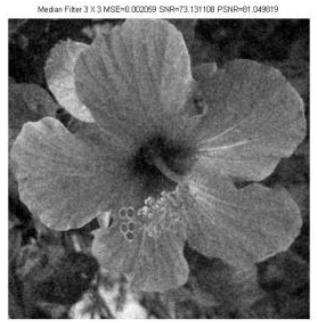

d

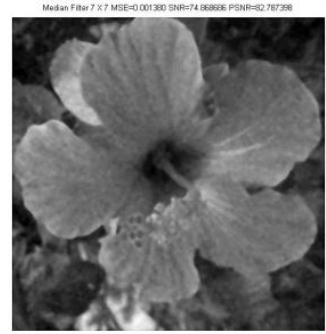

f 


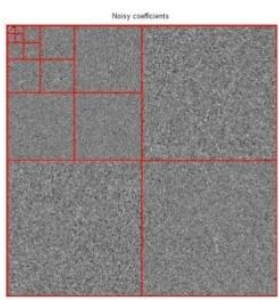

g

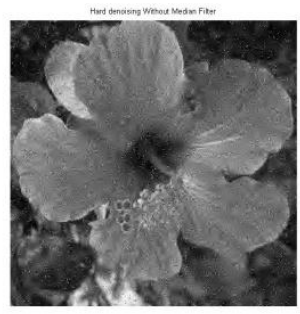

i

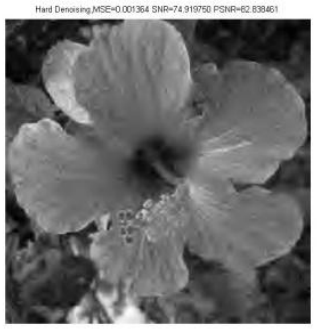

$\mathrm{k}$

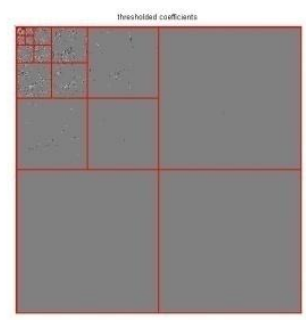

$\mathrm{h}$

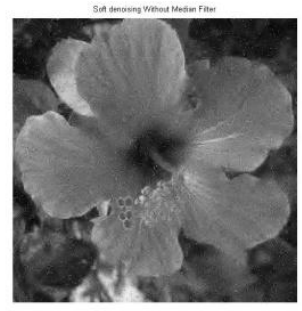

j

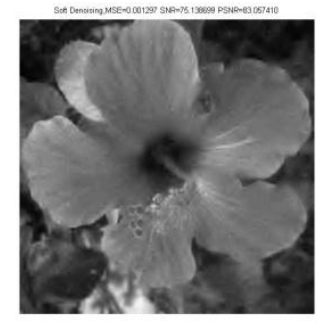

1
Figure 2: the above figure explain as follows:(a).Original image (b).Multilevel noisy image (c).Wiener filtered image (d). 3 X 3 median filtered image (e). $5 \times 5$ median filtered image (f). 7 X 7 median filter image (g).Noisy coefficient (h).Threshold coefficient(i).Hard filtered image without median filter (j).Soft filtered image without median filtered (k).Hard filtered image with median filtered (l).Soft filtered image with median filtered.

Table 1: Comparison of MSE ,SNR and PSNR values for multilevel noise.

\begin{tabular}{|c|c|c|c|}
\hline IMAGE & $\begin{array}{l}\text { Mean- } \\
\text { Square } \\
\text { Error( } \\
\text { MSE) }\end{array}$ & $\begin{array}{l}\text { Signal-to- } \\
\text { Noise } \\
\text { Ratio(SNR) }\end{array}$ & $\begin{array}{l}\text { Peak Signal- } \\
\text { to-Noise } \\
\text { Ratio(PSNR) }\end{array}$ \\
\hline $\begin{array}{l}\text { Multi-level } \\
\text { noise image }\end{array}$ & $\begin{array}{l}0.01544 \\
3\end{array}$ & 64.412713 & 72.331424 \\
\hline $\begin{array}{l}\text { Wiener Filter } \\
\text { Image }\end{array}$ & $\begin{array}{l}0.00506 \\
5\end{array}$ & 69.221228 & 77.139939 \\
\hline $\begin{array}{l}\text { Median } \\
\text { Filter } 3 \text { X } 3 \\
\text { image }\end{array}$ & $\begin{array}{l}0.00205 \\
9\end{array}$ & 73.131108 & 81.049819 \\
\hline $\begin{array}{l}\text { Median } \\
\text { Filter } 5 \text { X } 5 \\
\text { image }\end{array}$ & $\begin{array}{l}0.00131 \\
1\end{array}$ & 75.090509 & 83.009220 \\
\hline $\begin{array}{l}\text { Median } \\
\text { Filter } 7 \text { X7 } \\
\text { image }\end{array}$ & $\begin{array}{l}0.00138 \\
0\end{array}$ & 74.868686 & 82.787398 \\
\hline
\end{tabular}

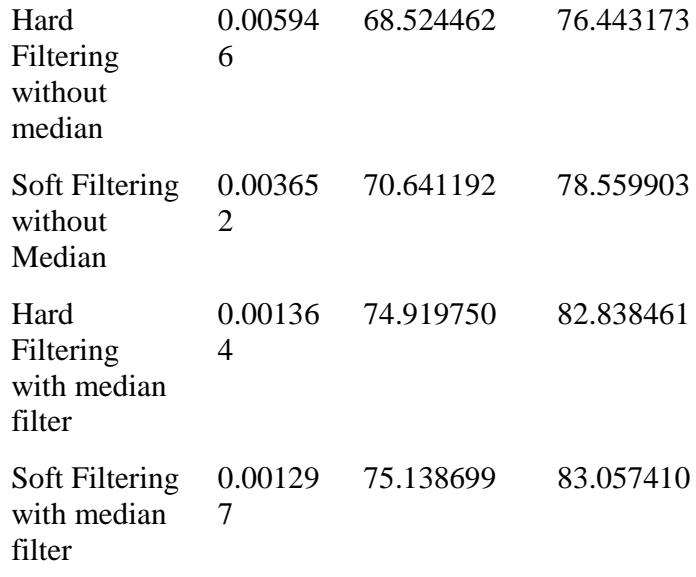

The above table show that median filter based wavelet transform method is more efficient for removing multilevel noise than other filters.

\section{CONCLUSION}

In this paper efficient techniques for de-noising for the image has been proposed combined median filter and wavelet transform .This method is verified on the image where these image are corrupted by noise at different density .Experimental result show that the combined with median and wavelet transform method is more efficient for removing multilevel noise.

\section{REFERENCE}

[1] David L. Donoho and Iain M. Johnstone. Minimax estimation via wavelet shrinkage.Technical report, 1992. 3.

[2] David L. Donoho and Jain M. Johnstone. Ideal spatial adaptation by wavelet shrinkage. Biometrika , 81(3):425\{455, 1994. 3, 10, 12, 13.

[3] David L. Donoho and Iain M. Johnstone. Adapting to unknown smoothness via wavelet shrinkage. Journal of the American Statistical Association, pages 1200\{1224, 1995. 3, 11, 13, 15, 24.

[4] David L. Donoho, Iain M. Johnstone, Gerard Kerkyacharian, and Dominique Picard.Wavelet shrinkage: asymptopia. Journal of the Royal Statistical Society, Ser. B, pages $371\{394,1995.3$

[5] David L. Donoho. De-noising by soft-thresholding. IEEE Transactions on Information Theory, 41(3):613 \{627, May 1995. 3, 24.

[6] Anestis Antoniadis, Jeremie Bigot, and Theofanis Sapatinas. Wavelet estimators in nonparametric regression: A comparative simulation study. Journal of Statistical Software,6(6):1,\{83, June 2001. 3, 20, 24.

[7] S. O. Rice "Mathematical analysis of random noise", Bell Syst. Tech. J., vol. 23, no. 3, pp.282 -332 1944.

[8] Y. H. Lee and S. A. Kassam "Generalized median filtering and related nonlinear filtering techniques", IEEE Trans. Acoust., Speech, Signal Processing, vol. ASSP-33, pp.672 -683 1985. 